\title{
Barrages de correction torrentielle : estimation des poussées de berges
}

\section{F. NICOT \\ J.-M. TACNET \\ Cemagref- Unité} de Recherche ETNA

2, rue de la Papeterie BP 7

38402 Saint-Martin-d'Hères francois.nicot@grenoble.cema gref.fr jean-

marc.tacnet@grenoble.cemagref.fr

\section{E. FLAVIGNY}

Laboratoire Sols, Solides,

Structures

Domaine universitaire

BP 53

38041 Grenoble Cedex 9 etienne.flavigny@hmg.inpg.fr
Les barrages de consolidation visent à limiter l'érosion du chenal d'écoulement des torrents et sont souvent implantés dans des sites oủ les berges sont fortement instables. Ces zones constituent en effet d'importantes zones d'alimentation en matériaux solides. Les méthodes actuelles de dimensionnement de ces ouvrages restent basées sur une approche bi-dimensionnelle d'estimation des actions et des réactions du sol en fondation. De même, les actions dues aux poussées des berges instables, qui entraînent de nombreuses pathologies sur les ouvrages, restent mal connues et ne sont pas prises en compte dans les justifications de la stabilité externe et interne des ouvrages. Cet article présente la problématique et la démarche d'étude en vue d'améliorer la connaissance des interactions entre les barrages et le sol au niveau des berges. Dans cette perspective, une approche analytique est proposée, puis les bases d'une simulation numérique introduisant la méthode des Éléments Finis sont définies.

Mots-clés : barrage de correction torrentielle, glissements de terrains, interaction sol/structure, mécanique des sols, méthode des éléments finis.

\section{Torrent control dams : assessment of the thrust of the banks}

Mitigation strategies against natural risks in mountainous areas often include protection works against torrential flooding. avalanches or falling rocks. These structures, which are submitted to specific phenomena as well as they are built in a difficult geotechnical context, must be designed in compliance with specific requirements, Torrent control dams based on an active protection concept are often built to limit the risks of bank slip in the upper part of the catchment area. Usually, design of such structures is based on a two-dimensional approach, allowing force reactions applied by the soil to the foundations of the structure to be assessed. Furthermore, the thrust of the bank is seldorn taken into account, even if the loading is likely to be the cause of a lot of observed damages. This paper exposes a methodology whose purpose is to improve the modelling of interaction between soil and structure. After an original analytical approach is presented, the main basis of numerical simulation (Finite Element Code) are defined.

Key words : torrent control dams, landslides, interaction soil/structures, solis Mechanics, finite element method. 


\section{Introduction}

Les matériaux transportés par les torrents en crue par charriage ou sous forme de laves torrentielles sont fréquemment issus des berges en glissement. Pour limiter les volumes de matériaux susceptibles de transiter vers l'aval, la construction de barrages de « consolidation » dans ces zones de berges instables présente un intérêt stratégique important dans un dispositif de correction torrentielle. Implantés en série dans les tronçons fortement érodables du chenal d'écoulement dans le cadre de travaux de correction active, ces ouvrages visent à limiter l'érosion longitudinale du lit du torrent, les divagations latérales des écoulements, l'érosion des berges et dans une moindre mesure laminer les laves torrentielles. Ces structures, en béton armé pour la plupart, ont une hauteur moyenne entre 5 et 15 mètres au niveau de la cuvette de déversoir et les plus courantes s'apparentent globalement à des murs de soutènement de type autostable (cantilever). La difficulté d'accès aux sites d'implantation des ouvrages limite souvent les possibilités d'emploi de moyens lourds pour leur réalisation. Les terrains de fondation sont souvent médiocres, fortement hétérogènes et de forte granulométrie (matériaux altérés, moraines à gros éléments, etc.).

En termes de dimensionnement, ces ouvrages se distinguent d'ouvrages d'art plus courants par la nature des charges agissant (chocs, poussée de lave torrentielle) et par un contexte géotechnique de mise en place difficile (caractéristiques géomécaniques du sol de fondation mal connues, instabilité de berges avec une forte interaction sol-structure, etc.). Ces ouvrages sont typiquement tridimensionnels en raison d'une géométrie fortement variable entre l'axe de l'ouvrage et les rives, et d'actions (poussée des terres, laves torrentielles) agissant non seulement dans des plans perpendiculaires au voile de l'ouvrage mais aussi dans des directions quelconques notamment dans le cas d'instabilité de berges. Des pathologies observées sur des barrages endommagés mettent en évidence la complexité de la détermination des efforts (cirection, intensité, répartition de contraintes) liés à l'effet de la poussée des berges sur ce type de structures (Fig. 1). Les matériaux

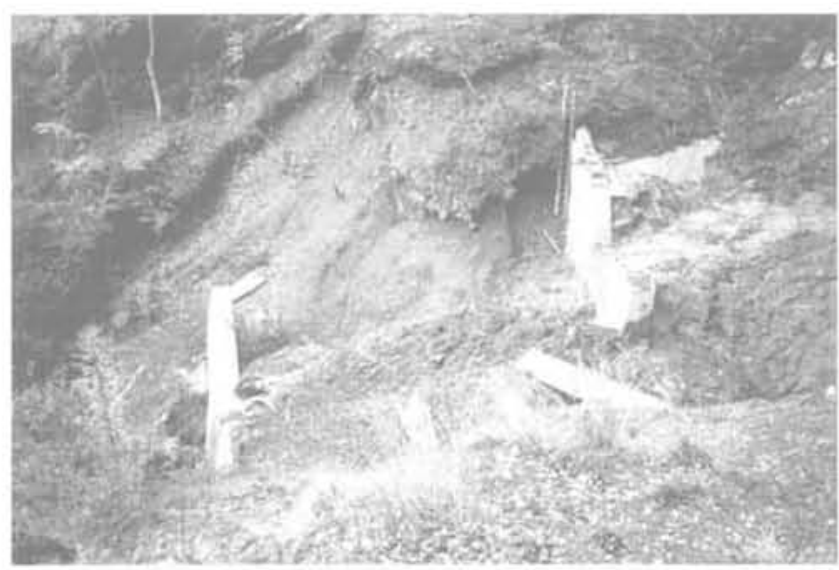

FIG.1 Action d'une berge instable sur un barrage de correction torrentielle de type consolidation.

Torrent control dam submitted to the thrust of an unsteady bank. rencontrés provenant d'éboulis de pentes sont souvent très hétérogènes et les conditions de site rendent les sondages ou essais in situ inadéquats; voir, par exemple, Gotteland et al. (2000) ; Tacnet et al. (2000). Dans le cas de pentes instables, on se trouve en présence de glissement dont à la fois la profondeur et la longueur de la zone active sont difficiles à déterminer. Très peu de travaux ont été consacrés jusqu'à présent à ce thème. Les quelques contributions existantes relèvent essentiellement du domaine de l'ingénierie; elles sont souvent basées sur des approches sommaires qui ne prennent pas - ou mal - en compte l'interaction mécanique entre les berges et l'ouvrage ; Deymier et al. (1995). Cet article présente un travail de recherche récemment mené au Cemagref, en collaboration avec le laboratoire 3S de Grenoble. Ce travail a eu pour objectifs d'identifier puis d'évaluer les actions dues à un glissement sur un barrage de correction torrentielle, de manière à optimiser par la suite la conception et le dimensionnement de ces structures.

Dans cette perspective, après avoir décrit les principales pathologies observées sur les barrages de correction torrentielle, une méthode analytique s'appuyant sur les travaux de Blondeau et Virollet (1976) est proposée ; cette méthode permet, dans le cas de la présence à faible profondeur d'un substratum rocheux parallèle à la pente, d'évaluer les efforts appliqués par les berges sur les parements latéraux de louvrage. La dernière partie est consacrée à l'application de la méthode des éléments finis. En particulier, les difficultés de mise en place de ce type de modélisation, dans un contexte d'instabilités de berges, sont évoquées. Enfin, une méthodologie pour le dimensionnement des barrages de correction torrentielle est dégagée.

\section{2}

\section{Quelques exemples de pathologies observées}

Les barrages de correction torrentielle, compte tenu de leur environnement géotechnique très spécifique, sont soumis aux actions suivantes :

- actions dues au torrent (fluide dont la rhéologie peut être complexe : matrice boueuse, pouvant inclure des blocs rocheux);

- actions dues aux berges latérales.

Si les actions dues au torrent sont de type dynamique, les secondes sont au contraire de type quasi statique. Néanmoins, leur estimation demeure aujourd'hui un problème ouvert.

Parmi les pathologies observées sur les barrages, certaines peuvent être imputées à l'action du fluide, et des éléments solides charriés ; mais la plupart doivent être cependant reliées à l'action des berges latérales, souvent dissymétrique, et orientée dans un plan différent de celui du barrage. L'action des berges conduit à des désordres qui sont de deux sortes:

- déplacements de corps rigide de l'ouvrage, par rapport aux berges (stabilité externe de l'ouvrage). On peut observer alors un basculement ou un enfoncement du barrage, parfois même un déplacement ou un basculement vers l'aval ;

- apparition d'un réseau de fissures plus ou moins ouvertes (stabilité interne de l'ouvrage). Ces fissures sont souvent orientées à 45 degrés par rapport à la 
direction horizontale (Fig. 2). Parfois, un réseau de fissures sub-horizontales s'observe également.

Dans certains cas, les deux types de désordres coexistent sur un même ouvrage : le barrage a subi un déplacement de corps rigide, auquel s'associe un système de fissures plus ou moins étendues. Lorsque la sollicitation est suffisamment importante, il peut y avoir rupture et déplacement d'une partie du barrage (Fig. 1).

Cet aperçu rapide suffit à démontrer les défauts de conception et de dimensionnement dont pâtissent ces ouvrages. Il souligne par ailleurs, à travers la variété des désordres observés, la complexité des mécanismes mis en jeu. Même si le coût de construction d'un barrage de correction reste faible, il importe de bien mesurer les conséquences économiques qui seraient induites plus en aval (présence d'enjeux) par la rupture d'un ou plusieurs ouvrages. Cette prise de conscience justifie que soit entreprise aujourd'hui une action de recherche visant à améliorer la conception et le dimensionnement de ces ouvrages.

\section{3}

\section{Approche analytique}

Cette section a pour objectif d'évaluer, à travers une approche bi-dimensionnelle simple et rapide de mise en œuvre, la résultante des efforts appliqués par un massif instable sur le parement d'un barrage de correction. Après avoir déterminé la valeur de la résultante qui assure l'équilibre du massif, la recherche d'un champ de contrainte licite au voisinage du parement fournira une relation supplémentaire portant sur la longueur du glissement.

\section{1}

\section{Contexte géomécanique}

On s'intéresse à l'action des berges dans le plan de l'ouvrage perpendiculaire à l'axe du torrent. Les berges sont représentées par une pente uniforme de longueur $L$, d'angle $\theta$, limitée en profondeur par la présence d'un substratum parallèle à la pente et situé à une profondeur $h$ (Fig. 3). On admettra par ailleurs que l'angle de
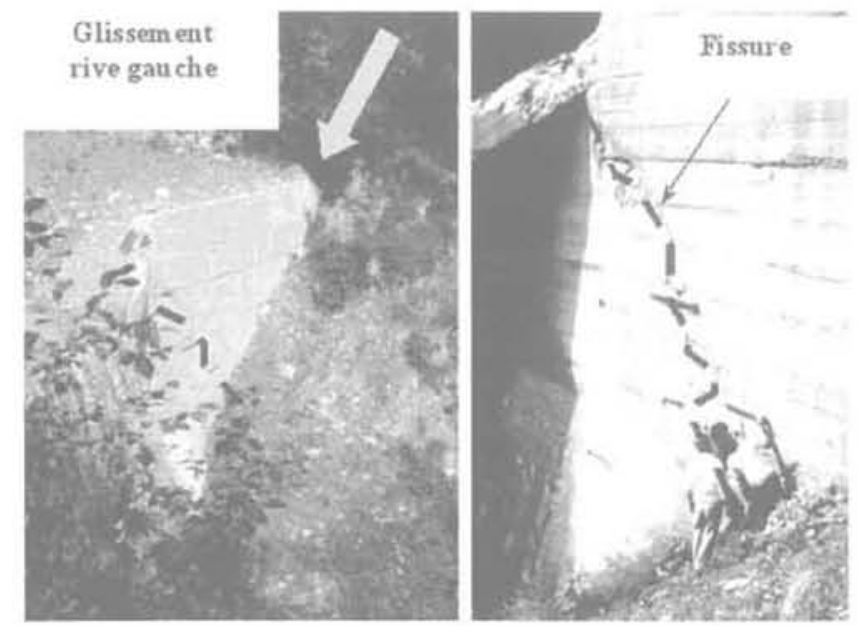

FQ. Q Quelques exemples de fissuration. Examples of fissures. la pente $\theta$ est supérieur à l'angle de frottement $\varphi$, et on supposera en première approximation qu'il n'y a pas d'écoulement d'eau au sein du massif. Dans un tel contexte géotechnique, le substratum apparait comme un plan de discontinuité brutale, susceptible de faciliter le glissement plan vers l'aval des matériaux sus-jacents. En outre, on se limitera dans le cadre de l'approche analytique au cas d'un ouvrage fondé dans le substratum rocheux supposé parfaitement rigide. De cette façon, seule l'action des berges sur le parement latéral de l'ouvrage, liée à la mobilisation des matériaux reposant au-dessus du substratum, sera analysée.

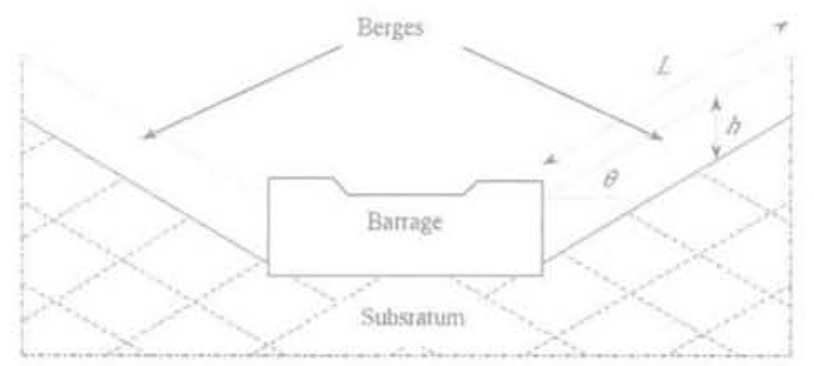

HG.3 Coupe schématique du barrage et des berges.

Schematic section of the dam with the two banks.

\section{2}

\section{Équilibre mécanique du massif de sol}

La réaction du parement appliquée sur le massif peut être déterminée en analysant l'équilibre mécanique de l'ensemble du massif, de longueur $L$, reposant sur le substratum (Fig. 4). On admettra que les contraintes le long de la surface de glissement DF sont uniformes, et on notera $N$ et $T$ leurs résultantes normale et tangentielle. En admettant que le sol est entré en plasticité le long de la surface de glissement, et en adoptant comme critère de plasticité le critère de Mohr-Coulomb, il vient alors :

$T=c L+N \tan \varphi$

où $c$ représente la cohésion du massif, et $\varphi$ l'angle de frottement, au niveau de la surface de glissement. En s'appuyant sur la figure 4, et en supposant que la surface EF reste libre, les équations d'équilibre suivantes sont déduites :

$$
\begin{aligned}
& X=\cos \theta\left[\gamma h L \cos ^{2} \theta(\tan \theta-\tan \varphi)-c L\right] \\
& Z=\sin \theta\left[\gamma h L \cos ^{2} \theta(\tan \theta-\tan \varphi)-c L\right]
\end{aligned}
$$

L'effort nécessaire pour équilibrer le massif admet alors pour expression :

$F_{\text {eq }}=\gamma h L \cos ^{2} \theta(\tan \theta-\tan \varphi)-c L$

Cette expression fait apparaître une dépendance linéaire entre la résultante appliquée sur le parement et la longueur du glissement $L$ :

$F_{e q}=L f_{e q}(c, \varphi)$ 

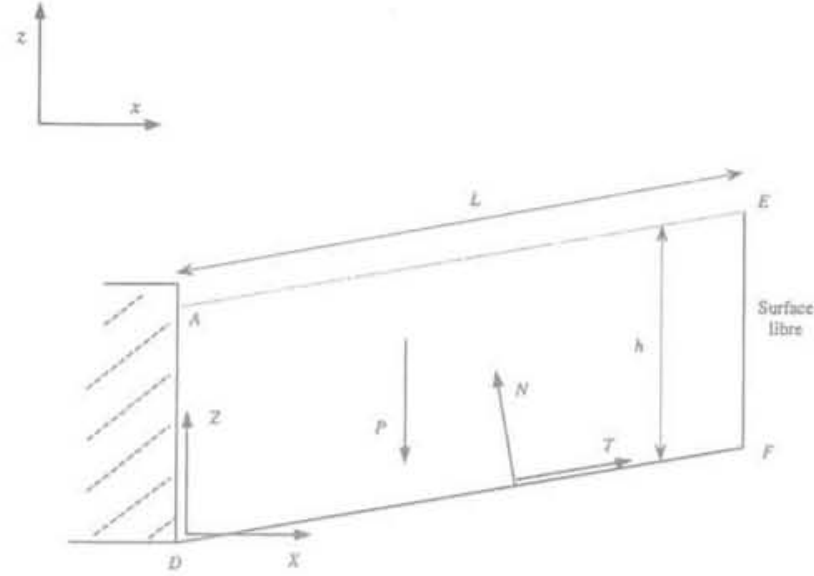

FiG.4 Équilibre mécanique du massif de sol. Mechanical balance of the bank.

\section{Analyse de la stabilité du massif de sol}

\section{3 .1}

\section{Étude d'un champ de contrainte au voisinage de l'ouvrage}

Si l'on admet que la contrainte s'exerçant sur une facette verticale est la même en tout point du massif situé à une même profondeur (ce qui revient à considérer le massif comme étant semi-infini), alors en un point $M$ du massif de sol situé à une profondeur $z$ ( $z<$ h), la contrainte sur une facette parallèle au substratum est donnée par les équations :

$\sigma_{0}(z)=\gamma z \cos ^{2} \theta$

$\tau_{0}(z)=\gamma z \cos \theta \sin \theta$

où $\gamma$ représente le poids volumique du terrain et $\theta$ la pente du substratum.

Par ailleurs, on admet que le volume élémentaire de sol autour du point $M$ est dans un état d'équilibre limite supérieur de Rankine. En adoptant le critère de MohrCoulomb comme critère de plasticité, le cercle de Mohr associé à l'état de contrainte au point $M$ est donc tangent aux deux droites de Mohr-Coulomb. L'hypothèse selon laquelle $\theta>\varphi$ induit l'existence d'une surface critique située à une profondeur $z_{c}$ définie par :

$\tau_{0}\left(z_{c}\right)=-c-\sigma_{0}\left(z_{c}\right) \tan \varphi$

$c^{\prime}$ est-à-dire

$z_{c}=\frac{c}{\gamma \cos ^{2} \theta(\tan \theta-\tan \varphi)}$

Si $z_{<}<h$, létat de contrainte en un point $M$ situé en dessous de la surface critique $(z>z)$ est associé à un cercle de Mohr qui franchit les deux droites de MohrCoulomb (Fig. 5), Cela signifie que les relations (6) et (7) ne sont plus valides lorsque $z>z_{\text {f }}$ : sans la présence de louvrage en pied de talus, le glissement se produirait donc avant même qu'une surface critique ne puisse se développer au-dessus. On admettra que la présence de l'ouvrage permet le développement de cette surface critique, en modifiant l'état de contrainte au sein du

massif de la façon suivante : la contrainte tangentielle $\tau_{\circ}^{*}(z)$ sur une facette parallèle au substratum est telle que le cercle de Mohr associé est tangent aux deux droites de Mohr-Coulomb (Fig. 5) ; Sanglerat et al. (1981). Ainsi, il vient:

$$
\begin{aligned}
& \sigma_{o}(z)=\gamma z \cos ^{2} \theta \\
& \tau_{o}^{*}(z)=-c-\sigma_{o}(z) \tan \varphi
\end{aligned}
$$

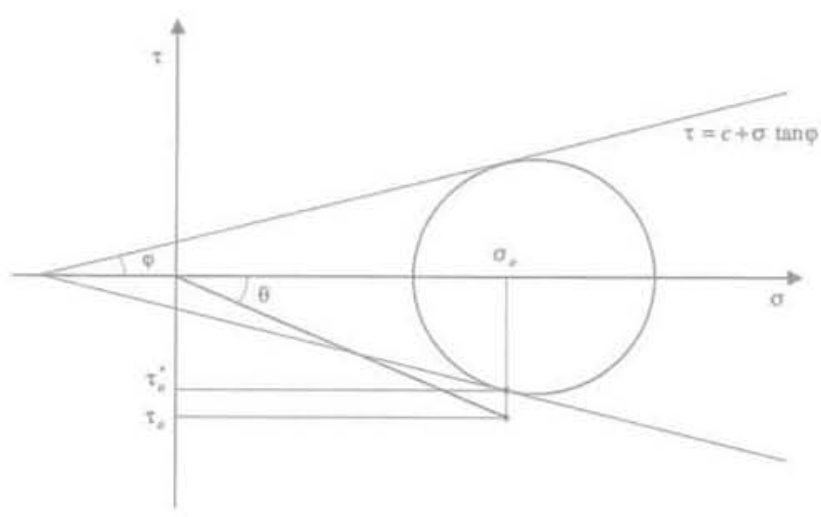

AG.5 Cercle de Mohr associé à l'état de contrainte au sein du massif de sol.

Mohr circle related to the state of stress into the bank.

\section{3 .2}

\section{Schéma cinématique associé au massif}

A partir de l'analyse mécanique proposée précédemment, les directions de glissement peuvent être déduites en tout point du massif dans un état d'équilibre limite ; de cette façon, le schéma cinématique suivant peut être proposé (Fig. 6).

- la zone I est constituée d'un coin de sol en équilibre surabondant (élastique) ;

- la zone II est constituée de sol en équilibre limite de butée (équilibre supérieur de Rankine) ;

- la zone III est en équilibre limite, assuré par la pré. sence de l'ouvrage.

Les zones II et III sont séparées par la surface critique qui est située à la profondeur $z$. Il importe de noter que l'extension des zones Il et III' en amont de la

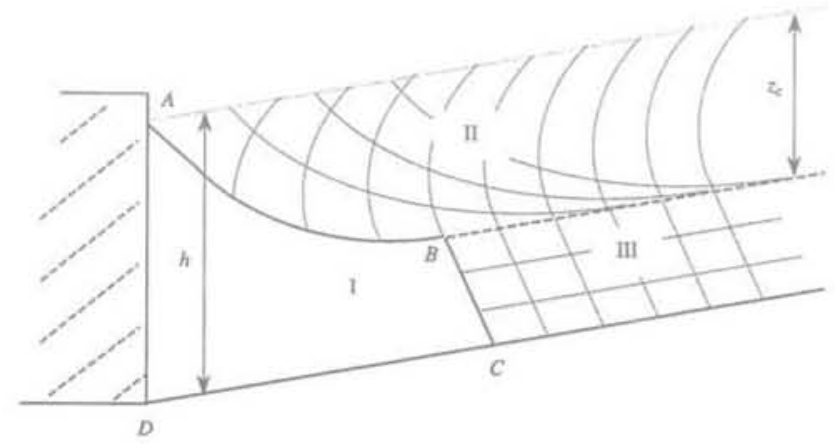

FG. 6 Schéma cinématique associé au massif de sol.

Kinematic scheme related to the bank. 
zone I est limitée. En effet, seule une partie du massif au voisinage de la zone I est en état d'équilibre limite. Au-delà, les expressions précédentes des contraintes normale et tangentielle (Eq. 6, 7 et 11) sont vraisemblablement incorrectes. En revanche, on peut admettre en première approximation que ces expressions demeurent valides le long de la frontière $A B C$. Ce résultat sera exploité dans le cadre de l'analyse mécanique du coin de sol $\mathrm{ABCD}$.

\section{3 .3}

\section{Équilibre mécanique du coin de sol en équilibre surabondant}

Afin d'évaluer le chargement imposé par le massif sur le parement de l'ouvrage, l'équilibre du coin de sol lié à l'ouvrage (zone I) est analysé. La géométrie de ce coin de sol n'est en fait pas complètement définie. En effet, la ligne de glissement issue du point $A$ admet pour asymptote la surface critique (profondeur $z$ ) ; il n'est donc pas possible de siturer la position du point $B$ le long de cette surface. De manière à simplifier les calculs, on adoptera l'hypothèse que ce coin de sol peut être assimilé à un quadrilatère $\mathrm{ABCD}$ (Fig. 7), dont l'arête $\mathrm{AB}$ est tangente au point $\mathrm{A}$ à la première ligne de glissement issue de ce point. De cette façon, l'angle $\alpha$ est choisi égal à $\left(\frac{\pi}{4}-\frac{\varphi}{2}\right)$. Le volume ABCD est soumis aux efforts suivants (Fig. 7) :

- son poids propre $P_{t}$;

- une distribution de contrainte $(\sigma, \tau)$ sur AB dont la résultante $F_{f}$ a pour composantes $\left(N_{\gamma}, T_{1}\right)$;

- une distribution de contrainte $\left(\sigma_{2}, \tau_{2}\right)$ sur BC dont la résultante $F_{2}$ a pour composantes $\left(N_{2}, T_{2}\right)$;

- une distribution de contrainte $\left(\sigma_{3}, \tau_{3}\right)$ sur CD dont la résultante $F_{3}$ a pour composantes $\left(N_{3}, T_{3}\right)$;

- la réaction du parement dont la résultante $F_{\text {lim }}$ a pour composantes $(X, Z)$.

\section{- Calcul de $N_{1}$ et $T_{1}$}

En prenant avantage de la construction du pôle (point $P$ sur la figure 8), il est aisé d'établir les expressions de $\sigma_{1}$ et $\tau_{1}$ (Costet, 1976) :

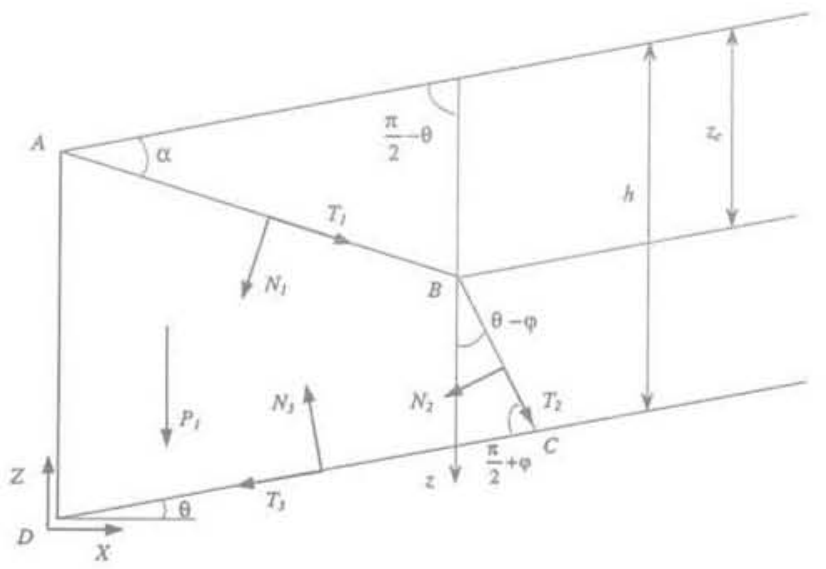

FG.7 Équilibre mécanique du quadrilatère ABCD.

Mechanical balance of quadrilateral $A B C D$. $\sigma_{1}=x-r \cos (\omega+2 \alpha)$

$\tau_{1}=-r \sin (\omega+2 \alpha)$

dans lesquelles:

$$
\begin{aligned}
& x=\frac{c+\sigma_{0} \tan \varphi+\sin \varphi \sqrt{\left(c+\sigma_{0} \tan \varphi\right)^{2}-\tau_{0}^{2}}}{-\frac{c}{\tan \varphi}}
\end{aligned}
$$

$$
\begin{aligned}
& T=x \sin \varphi+c \cos \varphi \\
& \tan \omega=\frac{\left|\tau_{0}\right|}{x-\sigma_{0}}
\end{aligned}
$$

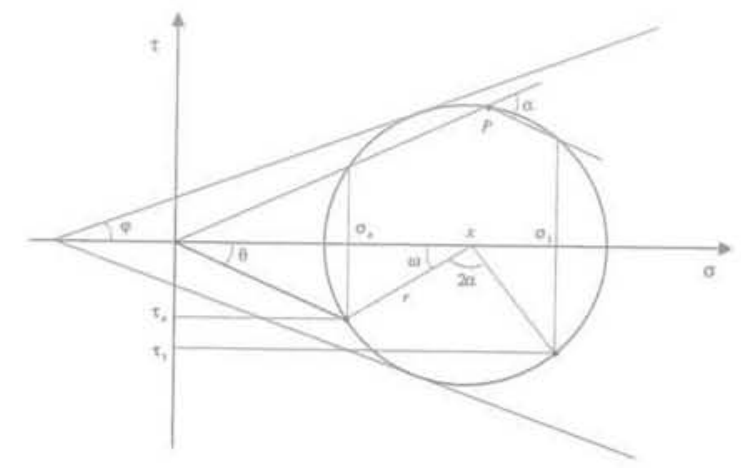

FG.8 Cercle de Mohr associé à l'état de contrainte sur $\mathrm{AB}$.

Mohr circle related to the state of stress along $\mathrm{AB}$.

Les expressions de $N_{1}$ et $T_{1}$ sont alors les suivantes :

$$
\begin{aligned}
& N_{1}=\int_{0}^{z_{c}} \sigma_{1}(z) \frac{\cos \theta}{\sin \alpha} d z \\
& I_{2}=\int_{0}^{z_{5}} \tau_{1}(z) \frac{\cos \theta}{\sin \alpha} d z
\end{aligned}
$$

\section{- Calcul de $\mathrm{N}_{2}$ et $T_{2}$}

Les équations 12 à 16 restent valides, en appliquant formellement $\tau_{o}(z)=-c-\sigma_{o}(z) \tan \varphi$.

Les expressions de $\mathrm{N}_{2}$ et $T_{2}$ sont alors les suivantes :

$$
\begin{aligned}
& N_{2}=\int_{z_{c}}^{h} \sigma_{2}(z) \frac{\cos \theta}{\sin \varphi} d z \\
& T_{2}=\int_{z_{c}}^{h} \tau_{2}(z) \frac{\cos \theta}{\cos \varphi} d z
\end{aligned}
$$

\section{- Calcul de $\mathrm{N}_{3}$ et $\mathrm{T}_{3}$}

Les contraintes $\left(\sigma_{3}, \tau_{3}\right)$, que l'on supposera uniformes le long du substratum, sont données par : 
$\sigma_{3}=\sigma_{\mathrm{o}}(h)$

$\tau_{3}=\tau_{0}^{*}(h)$

De cette façon, il vient :

$N_{3}=\gamma h C D \cos ^{2} \theta$

$T_{3}=C D\left(c+\gamma h \cos ^{2} \theta \tan \varphi\right)$

avec

$$
C D=z_{c} \frac{\cos (\alpha-\theta)}{\sin \alpha}-\left(h-z_{c}\right) \frac{\sin (\theta-\varphi)}{\cos \varphi}
$$

L'équilibre du massif de sol $A B C D$ fournit alors les deux relations :

$X=N_{1} \sin (\alpha-\theta)-T_{1} \cos (\alpha-\theta)+N_{2} \cos (\theta-\varphi)-\ldots$

$\ldots-T_{2} \sin (\theta-\varphi)+N_{3} \sin \theta+T_{3} \cos \theta$

$Z=N_{1} \cos (\alpha-\theta)+T_{1} \sin (\alpha-\theta)+N_{2} \sin (\theta-\varphi)+\ldots$

$\ldots+T_{2} \cos (\theta-\varphi)-N_{3} \cos \theta+T_{3} \sin \theta+P_{1}$

ce qui permet de définir la résultante $F_{\text {lim }}$ appliquée par le massif le parement latéral de l'ouvrage :

$$
F_{\text {lim }}=\sqrt{X^{2}+Z^{2}}=f_{\text {lim }}(c, \varphi)
$$

Contrairement à l'expression de $F_{\text {eq }}$ l'expression de $F_{\text {lim }}$ ne fait pas apparaitre de dépendance avec la longueur du glissement. $F_{\text {lim }} s^{\prime}$ interprète comme la valeur de la résultante appliquée au parement compatible avec un champ de contrainte licite sur la frontière amont du coin de sol $\mathrm{ABCD}$. C'est donc la valeur maximale de résultante qui peut être appliquée sur le parement.

\section{3 .4}

\section{Longueur critique de glissement}

La longueur critique de glissement $L$ est définie comme la longueur permettant d'assurer l'égalité $F_{\text {eq }}=$ $F_{\text {lim. }}$. La longueur critique apparaît donc comme un paramètre pertinent pour évaluer la stabilité du massif:

\section{Si $L<L \quad$ La stabilité du massif est assurée, l'effort} appliqué sur le parement est égal à $F_{\text {eq }}$.

Si $L=L \quad$ Le massif est, dans le voisinage du parement, en état d'équilibre limite. L'effort appliqué sur le parement est égal à $F_{\text {lim }}$ :

Si $L>L_{c} \quad$ L'état d'équilibre du massif n'est plus compatible avec un champ de contrainte licite. Le glissement est susceptible de se produire et de se déverser au-dessus du parement de l'ouvrage. L'effort appliqué sur le parement est borné par $F_{\text {lim }}$.

Pour un site donné, dont on connait les valeurs de $\gamma$, $h$ et $\theta, L_{c}$ est fonction de $c$ et $\varphi: L_{c}=f(c, \varphi)$. On considère le cas où $\theta=25$ degrés, $h=8$ mètres et $\gamma=18 \mathrm{kN} / \mathrm{m}^{3}$. La figure 9 représente l'évolution de $L_{c}$ en fonction de l'angle de frottement, pour différentes valeurs de cohésion. La longueur critique de glissement augmente avec $c$ et $\varphi$, et ce dautant plus vite que la valeur de $\varphi$ est élevée.

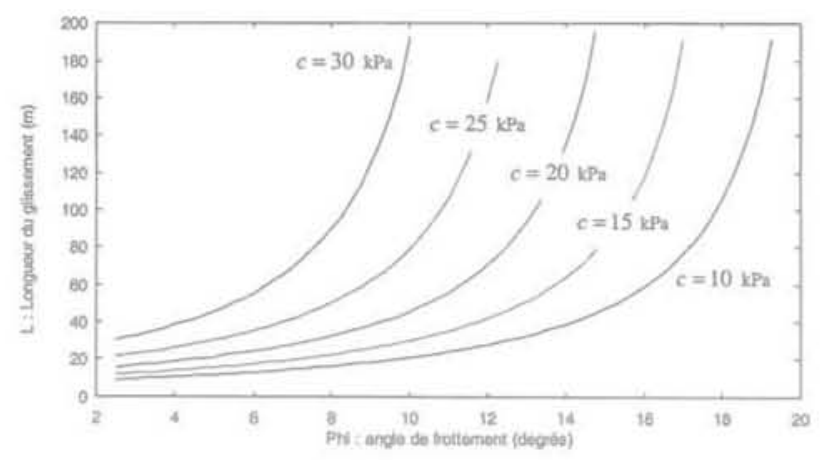

FG.9 Évolution de la longueur critique du glissement en fonction de $c$ et $\varphi$.

Evolution of the critical length of the lanslide as a function of both $c$ and $\varphi$.

\section{Sensibilité de la résultante aux paramètres mécaniques et géométriques}

En conservant les valeurs précédentes de paramètres : $\theta=25$ degrés, $H=8$ mètres et $\gamma=18 \mathrm{kN} / \mathrm{m}^{3}$, on examine successivement l'évolution de $F_{\text {lim }}$ en fonction de $L c$ pour différentes valeurs de cohésion (Fig. 10), puis l'évolution de $F_{\text {ium }}$ en fonction de $\varphi$ pour différentes valeurs de $L_{c}$ (Fig. 11).

La figure 10 montre une dépendance non-linéaire forte entre $F_{\text {lim }}$ et la longueur critique du glissement $L$. En revanche, pour une même longueur critique de glissement, on observe sur la figure 11 une faible dépendance entre $F_{\text {lin }}$ et les paramètres mécaniques $c$ et $\varphi_{\text {, }}$ $F_{\text {imm }}$ étant maximum lorsque l'angle de frottement devient nul. Ce résultat est intéressant, en particulier lorsqu'il s'agit d'un site où il n'est pas aisé d'apprécier les paramètres mécaniques $c$ et $\varphi$. On pourra alors choisir $\varphi=0$, et faire l'hypothèse que le massif est en état d'équilibre limite; la cohésion c vérifie alors la relation implicite $L=f(c, \varphi=0)$, où $L$ est la longueur estimée du glissement. Cette simplification permet d'estimer l'effort maximal qui peut être appliqué sur le parement de l'ouvrage. D'une manière générale, la méthodologie applicable sur un site donné pour déter-

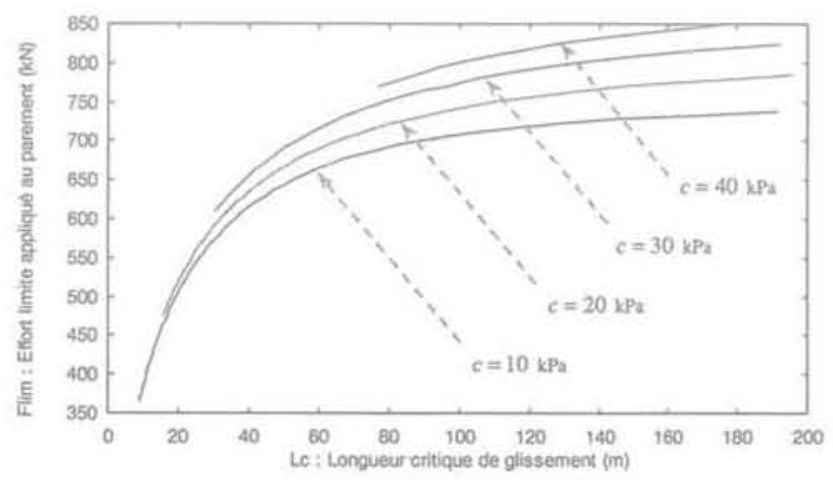

FG. 10 Évolution de $F_{\text {lim }}$ en fonction $L_{c}$, pour différentes valeurs de cohésion.

Evolution of $F_{\text {lim }}$ as a function of $L_{x}$, for several values of cohesion. 


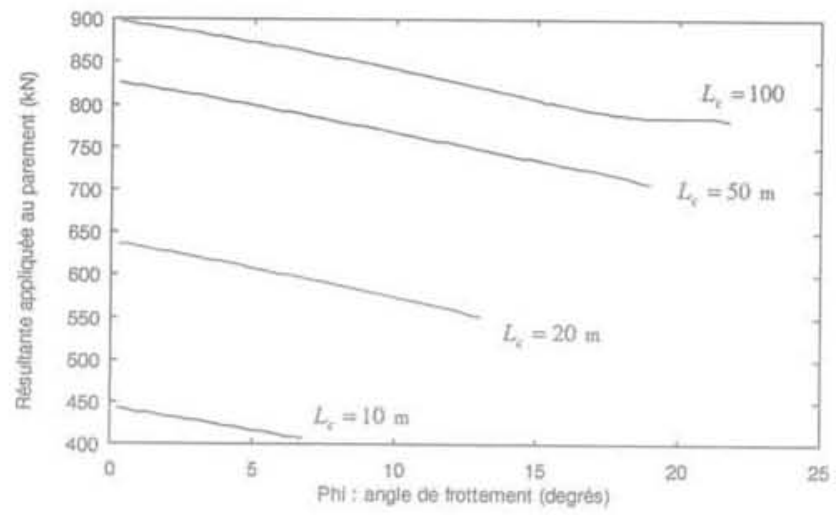

Fig.11 Évolution de $F_{\text {lim }}$ en fonction de l'angle de frottement pour différentes valeurs de $L$. Evolution of $F_{\text {lim }}$ as a function of the friction angle, for several values of $L$

TABLEAU Méthodologie d'estimation de l'effort appliqué sur l'ouvrage.

Assessment method of the force applied to the dam.

\begin{tabular}{|c|c|c|}
\hline & $c$ et $\varphi$ connus & $\begin{array}{c}\operatorname{cet} \varphi \\
\text { dificillement }\end{array}$ \\
\hline$L$ connue & $\begin{array}{l}F_{c q}=L f_{\mathrm{eq}}(c, \varphi) \\
F_{\text {limt }}=f_{\lim }(c, \varphi) \\
F=\min \left(F_{c q^{\prime}} F_{\mathrm{lim}}\right)\end{array}$ & $\begin{array}{l}\varphi=0 \text { et } c / L=f(c, \varphi=0) \\
F_{\lim }=f_{\lim }(c, \varphi) \\
F=F_{\lim }\end{array}$ \\
\hline $\begin{array}{l}L \text { difficilement } \\
\text { appréciable }\end{array}$ & $\begin{array}{l}L=f(c, \varphi) \\
F_{\lim }=f_{\text {iim }}(c, \varphi) \\
F=F_{\lim }\end{array}$ & \\
\hline
\end{tabular}

miner l'effort $F$ appliqué sur le parement de l'ouvrage est résumée sur le tableau I.

\section{5}

\section{Exemple d'application}

A titre d'illustration, on considère le cas d'une berge dont la longueur est difficilement appréciable. Le massif est constitué d'une argile remaniée, dont les paramètres sont :

$$
\begin{array}{ll}
\theta=25 \text { (degrés) } & \varphi=18 \text { (degrés) } \\
h=8(\text { mètres }) & c=10(\mathrm{kPa}) \\
\gamma=18\left(\mathrm{kN} / \mathrm{m}^{3}\right) &
\end{array}
$$

Afin de se placer dans les conditions les plus défavorables, on fait l'hypothèse que le massif est en état d'équilibre limite, ce qui conduit à une valeur de résultante $F=f_{\text {lim }}(c, \varphi)$. On obtient alors comme valeurs numériques pour $F$ et ses deux composantes $X$ et $Z$ :

$F=809(\mathrm{kN})$

$X=735(\mathrm{kN})$

$Z=338(\mathrm{kN})$
Avec de telles hypothèses, la longueur du glissement est égale à 100 mètres.

Il s'agit bien sûr d'une méthode très simplifiée, bidimensionnelle, et reposant sur plusieurs hypothèses ; mais elle est facile et rapide à mettre en œuvre, et peut fournir en particulier une première estimation de l'action de la poussée des berges dans le plan du barrage. Néanmoins, seule la résultante de cette action est évaluée; cette méthode ne permet pas de connaitre la distribution des contraintes le long du parement du barrage. Cette limitation justifie en partie l'introduction d’une approche numérique plus fine.

\section{4}

\section{Approche par la méthode des éléments finis}

En complément de l'approche analytique développée ci dessus, une série de modélisations numériques a été entreprise. Ces modélisations numériques, bidimensionnelles, sont réalisées sur une coupe perpendiculaire à l'axe du torrent : cette approche a été préférée à ce stade de l'étude à une modélisation tridimensionnelle.

Les objectifs de ces modélisations sont les suivants : - comparaison avec la méthode analytique :

- connaissance des efforts internes dans l'ouvrage : - évaluation du rôle de la géométrie de l'ouvrage ; - évaluation de l'effet de l'inclinaison des berges latérales:

- évaluation de l'effet d'une surface de glissement préexistante dans les berges.

\section{Modélisation}

Les modélisations ont été effectuées avec le logiciel Plaxis (Brinkgreve, 1998). Le comportement du sol est un modèle élastique-plastique de Mohr Coulomb sans écrouissage. Les caractéristiques géométriques et géotechniques sont identiques à celles retenues pour l'approche analytique, sauf les points suivants :

- la longueur de la pente a été réduite à 75 mètres. Une étude de sensibilité a montré que cela n'avait pas d'influence sur les résultats ultérieurs:

- un module d'Young pour le sol de $13 \mathrm{MPa}$ et un coefficient de Poisson de 0,3 ont été adoptés. Ces valeurs de paramètres élastiques ne sont pas nécessaires dans l'approche analytique en plasticité parfaite. Les valeurs de cohésion et d'angle de frottement sont respectivement égales à $10 \mathrm{kPa}$ et 18 degrés, identiques à celles retenues dans la section précédente.

Le maillage retenu est indiqué sur la figure 12 : une zone de substratum a été introduite sous la couche de sol. Une interface, sans réduction de propriétés mécaniques, permet le glissement de la couche de sol sur le substratum. On a choisi de ne pas réduire les propriétés mécaniques sur cette interface : elle est donc parfaitement rugueuse. Son rôle est de permettre le glissement entre la couche de sol et le substratum en créant ainsi des nœuds supplémentaires dans le 


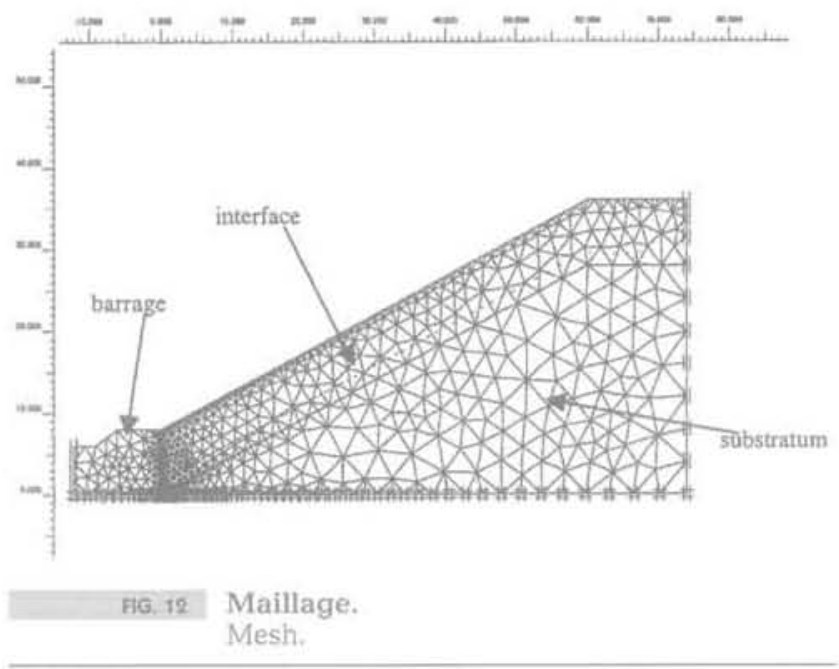

maillage, Le chargement du modèle a été mené en appliquant le poids propre à la couche de sol : un poids volumique de $18 \mathrm{kN} / \mathrm{m}^{3}$ a été considéré. Il n'a pas été pris en compte de nappe phréatique.

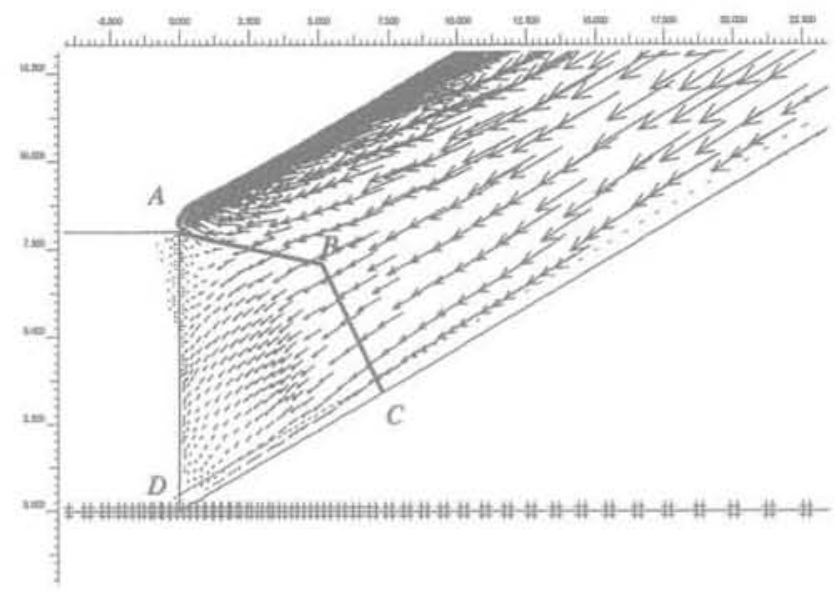

FIG. 13 Champ de déplacements incrémentaux. Displacement increments.

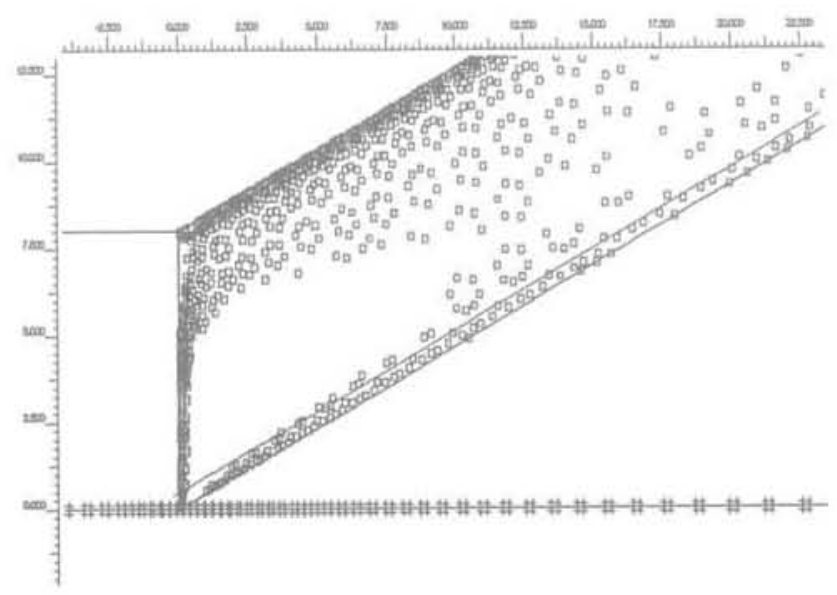

FiG. 14 Points plastiques. Plastic points.

\section{Résultats}

La modélisation par éléments finis permet de connaître les champs de déplacements et de contraintes en tout point du massif. Dans cette étude, on s'intéresse particulièrement à la cinématique de rupture. Le champ de déplacement incrémental représenté sur la figure 13 correspond alors aux vecteurs de déplacement entre les deux derniers pas de calcul. Il permet de visualiser l'écoulement plastique de la pente au-dessus du barrage, et aussi la zone où l'on retrouve un coin de sol $\mathrm{ABCD}$ analogue à celui mis en évidence sur la figure 6 . Un examen des points où le critère de plasticité est vérifié (Fig. 14) montre que le sol est plastifié sur l'interface, le long du parement $\mathrm{AD}$, et aussi en surface.

\section{3}

\section{Coefficient de sécurité}

Il est possible de calculer le coefficient de sécurité fs de la pente par une méthode analogue à la méthode de Taylor en diminuant cohésion et angle de frottement. Appliquée au cas présent, cette méthode fournit un coefficient de sécurité de l'ordre de 1.03. Les déplacements calculés n'ont alors pas de signification phy-

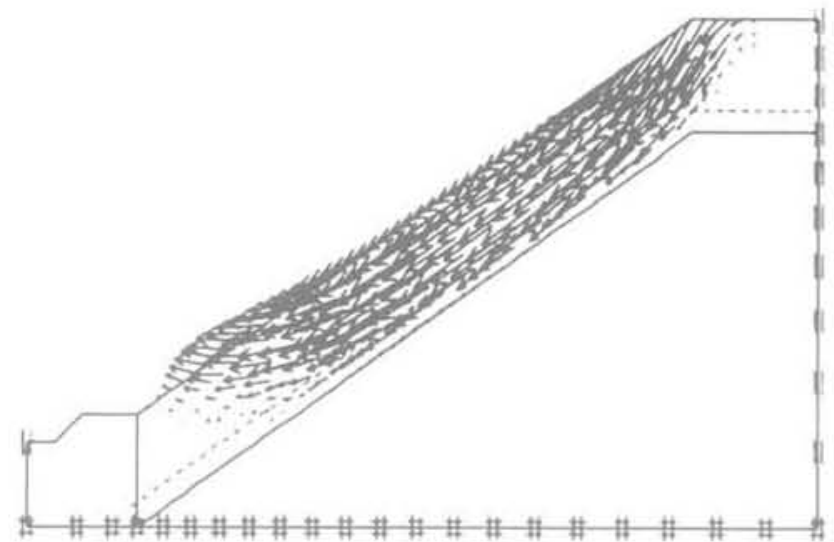

FlG.15 Champ de déplacement incrémental. Displacement increments.

sique, mais leur champ de vecteurs permet de figurer la forme de rupture qui se développe dans le massif de sol (Fig. 15). La valeur du coefficient de sécurité est légèrement supérieure à 1 : c'est pourquoi les calculs précédents ont pu être menés en appliquant l'intégralité du poids propre de la couche de sol.

\section{4}

\section{Efforts appliqués sur l'ouvrage}

Il est possible de calculer les efforts appliqués sur le parement vertical de l'ouvrage en intégrant les répartitions de contraintes normales et tangentielles agissant. 
Ce calcul fournit :

$F=717(\mathrm{kN})$

$\mathrm{X}=680(\mathrm{kN})$

$\mathrm{Z}=228(\mathrm{kN})$

Ces valeurs sont cohérentes avec celles obtenues par l'approche analytique. Les répartitions des contraintes sont données sur la figure 16. Il est important de noter que la répartition de contraintes normales n'augmente pas linéairement avec la profondeur: elle est même décroissante à partir de la mi-hauteur du parement; la partie haute de l'ouvrage est alors fortement sollicitée. La valeur maximale de la contrainte normale est de $140 \mathrm{kPa}$, et celle de la contrainte de cisaillement de $57 \mathrm{kPa}$ à une profondeur de 4,2 mètres sous la crête de l'ouvrage. Les irrégularités sur les
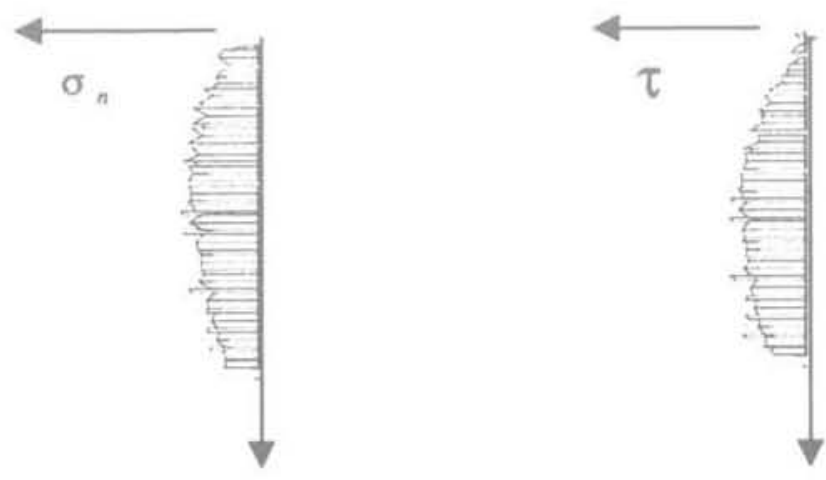

f16.16 Répartition des contraintes normale $\delta_{n}$ et tangentielle $\tau$ sur le parement.

Distribution of normal stress $\sigma_{n}$ and shear stress $\tau$ along the wall. courbes de contraintes (Fig. 16) correspondent au choix du maillage : un maillage plus fin aurait permis d'éviter cet effet parasite.

\section{5}

\section{Conclusion}

L'étude décrite dans cet article a permis de mettre en évidence l'intérêt d'une approche analytique bi-dimensionnelle. Il s'agit d'une approche simplifiée, mais qui permet d'estimer la longueur de glissement affectant les berges latérales, et la résultante des efforts appliqués par le massif sur le parement de l'ouvrage. Elle présente donc un intérêt certain pour l'ingénierie dans le cadre d'une phase de pré-dimensionnement des ouvrages de correction. La comparaison des résultats avec ceux fournis par une modélisation aux éléments finis a permis de constater une bonne cohérence entre les deux méthodes. Si la modélisation aux éléments finis fournit une information plus complète de l'action imposée sur l'ouvrage (distribution de contraintes le long du parement), il importe de souligner les difficultés de mise en œuvre de cette méthode dans un contexte d'instabilité de berges, liées aux déformations importantes que subit le massif au voisinage de son état d'équilibre limite.

Enfin, il convient de noter que la limitation majeure de la présente étude reste son caractère bi-dimensionnel. Les efforts mis en jeu peuvent être absorbés par la structure s'ils restent dans le plan du voile. Par contre, si les conditions géométriques de l'ouvrage par rapport à la pente entraînent des efforts hors plan, la flexion parasite ainsi créée est néfaste et nécessite alors des calculs tridimensionnels.

\section{Bibliographie}

Blondeau F., Virollet M. - * Les méthodes d'analyse de stabilité n. Bulletin de liaison des Laboratoires des ponts et chaussées nº spécial, 1967, p. 149-154.

Brinkgreve R.B.J., Vermeer P.A. - Plaxis, version 7, Rotterdam. Balkema, 1998.

Costet J., Sanglerat G. - Cours pratique de mécanique des sols. Éd. Dunod, 1976.

Deymier C., Tacnet J.M., Mathys N. Conception et calcul de barrages de cor- rection torrentielle. Etudes, $n^{\circ} 18$, Cemagref Éditions, 1995.

Gotteland P., Tacnet J.M. - « Caractérisation des sols grossiers, application aux sols de torrents ». Revue Française de Génie Civil, vol, $4, n^{\circ} 2-3,2000$, p. $328-338$.

Sanglerat G. Cambou B. Olivari G. - Problèmes pratiques de mécanique des sols et de fondation. Dunod, Tome 2. 1981. p. 216-257.
Tacnet J.M., Gotteland P. - « Mesure des caractéristiques géotechniques des sols grossiers, application aux sols de torrent $\gg$. Actes du colloque Inter-Praevent. Villach, Autriche, 2000.

Taylor D.W.- Fundamentals of Soil Mechanics. New York, John Wiley and sons, 1948. 